\title{
Endorectal Ultrasound-Guided Fine-Needle Aspiration: A Useful Diagnostic Tool for Perirectal and Intraluminal Lesions
}

\author{
Zahra Maleki $^{\mathrm{a}}$ Yener Erozan $^{\mathrm{a}}$ Susan Geddes $^{\mathrm{b}}$ Qing Kay Li ${ }^{\mathrm{b}}$

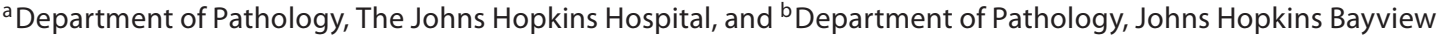 \\ Medical Center, Baltimore, Md., USA
}

\section{Key Words}

Endorectal endoscopic ultrasound $\cdot$ Fine-needle

aspiration $\cdot$ Colorectal adenocarcinoma

\begin{abstract}
Objectives: Endorectal endoscopic ultrasound (ERUS) allows highly detailed assessment of the rectal wall layers and visualization of the extraluminal structures. Herein, we study the utility of ERUS fine-needle aspiration (FNA) to evaluate perirectal lesions. Study Design: Forty-nine ERUS-FNAs were retrieved from the cytopathology archives of The Johns Hopkins Hospital. The cytology slides, corresponding histology, immunohistochemistry when available, and clinical data were reviewed. Results: The aspirated material showed malignant $(n=24)$, benign ( $n=19)$, atypical $(n=3)$, carcinoid tumor $(n=1)$, and nondiagnostic conditions $(n=2)$. The past medical history of 36 cases was significant for carcinomas. The primary site of the tumors included colorectal, urinary bladder, prostate, pancreas, gallbladder, ovary, and female lower genital tract. Statistical analysis for endoscopic ultrasonography FNA showed $87 \%$ sensitivity, 100\% specificity, diagnostic accuracy of $90 \%$, and a positive predictive value of $100 \%$ and a negative predictive value of $77 \%$. Conclusion: ERUS-FNA can be utilized for: (1) accurate staging of colorec-
\end{abstract}

tal adenocarcinomas by evaluation of nodal metastasis, depth of transmural tumor invasion and local tumor spread to perirectal fat, (2) prevention of aggressive surgical intervention in benign conditions, (3) providing diagnostic material for ancillary studies, and (4) evaluation of perirectal lesions with a more accurate method by combining imaging and histology.

Copyright $\odot 2012$ S. Karger AG, Basel

\section{Introduction}

Endoscopic ultrasonography (EUS) is a combination of endoscopy and intraluminal sonography. Due to the short distance to the target lesions, EUS yields high resolution images using a high frequency transducer. Endorectal endoscopic ultrasound (ERUS) represents one of the most significant developments in endoscopy in the last 20 years, and it is routinely used in daily clinical practice for diagnosis and staging of malignancies of the colorectal region and its surrounding structures such as

This paper was partially presented at the USCAP 2011 Annual Meeting in San Antonio, Tex., USA, February 26-March 4, 2011.

\section{KARGER \\ Fax +4161306 1234 \\ E-Mail karger@karger.ch}

www.karger.com
(C) $2012 \mathrm{~S}$. Karger AG, Basel

$0001-5547 / 13 / 0571-0009 \$ 38.00 / 0$

Accessible online at:

www.karger.com/acy
Correspondence to: Dr. Zahra Maleki

Department of Pathology, The Johns Hopkins Hospital $600 \mathrm{~N}$. Wolfe St.

Baltimore, MD 21287 (USA)

E-Mail Zmaleki1@jhmi.edu 
the prostate and bladder. ERUS allows highly detailed assessment of the rectal wall layers as well as visualization of the extra-luminal structures. It can be used to confirm malignancy in suspicious lesions as well as to identify and confirm nodal metastasis, tumor recurrence, or metastatic spread. Assessment of the depth of tumor penetration provides information for $\mathrm{T}$ staging in the TNM classification, and the ultrasonographic staging is suggested by the prefix ' $u$ ' [1]. For $\mathrm{N}$ staging, involvement is usually suspected if a lymph node is over $5 \mathrm{~mm}$ in diameter, round, and hypoechoic, features which may differentiate it from inflammatory nodes [2]. Furthermore, a tissue diagnosis of malignancy can be established by performing ERUS fine-needle aspiration (FNA) [3]. Herein, 49 ERUS-FNA specimens from 47 patients and their corresponding forceps biopsies or resected tissue specimens over a 22-year period have retrospectively been reviewed. ERUS-FNA findings have been reviewed to illustrate the utility of this relatively new technique in the diagnosis, staging, treatment, and prognosis of malignant neoplasms and the subsequent prevention of unnecessary aggressive surgical intervention in benign conditions.

\section{Materials and Methods}

Institutional Review Board approval for this study was obtained. The cytopathology archives of The Johns Hopkins Hospital were retrospectively searched for ERUS-FNA cases performed at this institution between January 1, 1990, and January 1,2012 . There were 49 perirectal FNA specimens from 47 patients obtained under ERUS guidance. EUS-FNAs were performed by gastroenterologists in an endoscopy unit or a radiologist in an ultrasound unit. A pathologist or cytotechnologist was present during the procedure for on-site evaluation of specimen adequacy when it was requested. Multiple aspirations were performed on each case. The aspirated material was immediately smeared on glass slides. The air-dried smears were stained with Diff-Quik stain for immediate on-site evaluation. Additional smeared slides were fixed in 95\% ethanol and stained with Papanicolaou stain. The remaining material in the needle was rinsed with Hank's balanced salt solution immediately after the procedure. The paraffin cell blocks were made of rinsed material and $4-\mu \mathrm{m}$ sections were stained with hematoxylin-eosin $(\mathrm{H} \& \mathrm{E})$. In addition to cell blocks, cytology slides were prepared using a liquid-based preparation method, e.g. cytospins. The number of passes, specimen adequacy on site, and the presence of a cytotechnologist or cytopathologist were recorded. Performance of the procedure by either a radiologist or a gastroenterologist and prophylactic administration of antibiotics was also noted. Immunohistochemical stains were done as needed on cell block material. On final cytopathologic examination 'unsatisfactory' diagnoses were rendered in cases in which the specimen was paucicellular. Perirectal FNA slides and their corresponding tissue biopsies or resections were reviewed by the authors and their cytology-histology diagnoses were correlated. Clinical information including patients' demographics, medical history including prior malignancy, endoscopy, cytology, and histology reports, as well as imaging studies and the patients' subsequent treatment and follow-up, were reviewed. The size of the mass lesions, the number of passes, and on-site adequacy interpretations were recorded and correlated.

\section{Results}

Forty-nine specimens from 47 patients of perirectal or intraluminal FNAs were reviewed. There were 22 males and 25 females (M:F ratio 0.88). Their ages ranged from 17 to 79 years $($ mean $=55.6$ years). Two patients had two specimens each with histologic confirmation. One patient had a perirectal lymph node and a soft tissue mass which were diagnosed as a benign lymph node and poorly differentiated squamous cell carcinoma, respectively. The second patient had a rectal nodule which was biopsied twice and diagnosed benign both times. The microscopic examination of aspirated material was noted as malignant $(n=24)$, benign $(n=19)$, markedly atypical cells suspicious for carcinoma $(\mathrm{n}=3)$, carcinoid tumor $(\mathrm{n}=1)$, and nondiagnostic $(\mathrm{n}=2)$.

Malignant cases (table 1) were colorectal adenocarcinoma $(n=9)$ (fig. 1), urothelial carcinoma $(n=64)$ (fig. $2)$, squamous cell carcinoma $(n=4)$, prostatic adenocarcinoma $(\mathrm{n}=1)$, metastatic adenocarcinoma of the lung $(\mathrm{n}=1)$, metastatic adenocarcinoma of the gallbladder $(\mathrm{n}=1)$, metastatic carcinoma of the breast $(\mathrm{n}=1)$, and metastatic carcinoma of the pancreas $(n=1)$. Twenty-one of 24 cases had concurrent core biopsy or surgical resection confirming the cytologic diagnosis of adenocarcinoma. One of 24 patients who had no history of carcinoma was diagnosed with metastatic poorly differentiated lung adenocarcinoma on ERUS-FNA. The patient had a large lung mass, multiple lytic lesions of bone, and a perirectal mass.

Immunohistochemical stains performed on cell blocks were available for 7 cases with malignant cytology. CK7, CK20, CDX2, PSA, PSAP, CEA, chromogranin, synaptophysin, and CD56 were commonly used immunostains. CK7, CK20, CDX2, and CEA are utilized to diagnose metastatic gallbladder carcinoma, pancreatic adenocarcinoma, and colonic adenocarcinoma. PSA and PSAP are used to differentiate prostatic adenocarcinoma, and neuroendocrine markers are used to differentiate neuroendocrine features in colonic adenocarcinoma and a poorly differentiated squamous cell carcinoma with basaloid features. 
Table 1. Cytologic findings of ERUS-FNA in patients with a known history of carcinoma

\begin{tabular}{|c|c|c|c|c|c|}
\hline $\begin{array}{l}\text { ERUS-FNA } \\
\text { diagnosis }\end{array}$ & $\begin{array}{l}\text { Histologic } \\
\text { diagnosis }\end{array}$ & History of cancer & $\begin{array}{l}\text { Imaging } \\
\text { diagnosis }\end{array}$ & $\begin{array}{l}\text { Clinical } \\
\text { significance }\end{array}$ & Change in treatment \\
\hline $\begin{array}{l}\text { Colorectal } \\
\text { adenocarcinoma }\end{array}$ & $\begin{array}{l}\text { Consistent with } \\
\text { histology }\end{array}$ & $\begin{array}{l}\text { Colorectal } \\
\text { adenocarcinoma }\end{array}$ & $\begin{array}{l}\mathrm{CT} / \text { hematoma, or } \\
\text { soft tissue }\end{array}$ & Metastasis & None \\
\hline $\begin{array}{l}\text { Colorectal } \\
\text { adenocarcinoma }\end{array}$ & $\begin{array}{l}\text { Colorectal } \\
\text { adenocarcinoma }\end{array}$ & $\begin{array}{l}\text { Colorectal } \\
\text { adenocarcinoma }\end{array}$ & $\begin{array}{l}\mathrm{CT} / \text { negative for } \\
\text { mass }\end{array}$ & N0 staging to N1 & Chemoradiation prior to surgery \\
\hline $\begin{array}{l}\text { Colorectal } \\
\text { adenocarcinoma }\end{array}$ & $\begin{array}{l}\text { Consistent } \\
\text { with histology }\end{array}$ & $\begin{array}{l}\text { Colorectal } \\
\text { adenocarcinoma }\end{array}$ & $\mathrm{CT} /$ negative & N0 staging to N1 & Chemoradiation prior to surgery \\
\hline $\begin{array}{l}\text { Colorectal } \\
\text { adenocarcinoma }\end{array}$ & $\begin{array}{l}\text { Colorectal } \\
\text { adenocarcinoma }\end{array}$ & $\begin{array}{l}\text { Colorectal } \\
\text { adenocarcinoma }\end{array}$ & $\begin{array}{l}\mathrm{CT} / \mathrm{mass} \text { favor } \\
\text { carcinoma }\end{array}$ & T3N0M0 & Chemoradiation prior to surgery \\
\hline $\begin{array}{l}\text { Colorectal } \\
\text { adenocarcinoma }\end{array}$ & $\begin{array}{l}\text { Colorectal } \\
\text { adenocarcinoma }\end{array}$ & $\begin{array}{l}\text { Colorectal } \\
\text { adenocarcinoma }\end{array}$ & Not available & First time Dx & Surgery followed by chemoradiation \\
\hline $\begin{array}{l}\text { Colorectal } \\
\text { adenocarcinoma }\end{array}$ & $\begin{array}{l}\text { Colorectal } \\
\text { adenocarcinoma }\end{array}$ & $\begin{array}{l}\text { Colorectal } \\
\text { adenocarcinoma }\end{array}$ & $\begin{array}{l}\text { MRI/colorectal } \\
\text { wall thickening }\end{array}$ & Recurrence & Surgery followed by chemoradiation \\
\hline $\begin{array}{l}\text { Colorectal } \\
\text { adenocarcinoma }\end{array}$ & $\begin{array}{l}\text { Colorectal } \\
\text { adenocarcinoma }\end{array}$ & $\begin{array}{l}\text { Colorectal } \\
\text { adenocarcinoma }\end{array}$ & $\begin{array}{l}\text { CT/recurrence, } \\
\text { soft tissue mass }\end{array}$ & Recurrence & Chemoradiation \\
\hline $\begin{array}{l}\text { Colorectal } \\
\text { adenocarcinoma }\end{array}$ & $\begin{array}{l}\text { Colorectal } \\
\text { adenocarcinoma }\end{array}$ & $\begin{array}{l}\text { Colorectal } \\
\text { adenocarcinoma }\end{array}$ & $\begin{array}{l}\text { CT/sarcoma or } \\
\text { GIST }\end{array}$ & $\begin{array}{l}\text { T2 staging } \\
\text { to } \mathrm{T} 3\end{array}$ & More radiation \\
\hline $\begin{array}{l}\text { Colorectal } \\
\text { adenocarcinoma }\end{array}$ & $\begin{array}{l}\text { Colorectal } \\
\text { adenocarcinoma }\end{array}$ & $\begin{array}{l}\text { Colorectal } \\
\text { adenocarcinoma }\end{array}$ & $\mathrm{CT} / \mathrm{metastasis}$ & Recurrence & Chemoradiation \\
\hline $\begin{array}{l}\text { Urothelial } \\
\text { carcinoma }\end{array}$ & $\begin{array}{l}\text { Consistent } \\
\text { with histology }\end{array}$ & $\begin{array}{l}\text { Urothelial } \\
\text { carcinoma }\end{array}$ & $\begin{array}{l}\mathrm{CT} / \text { metastasis or } \\
\text { recurrence }\end{array}$ & $\begin{array}{l}\text { Metastasis, } \\
\text { N0 to N2 }\end{array}$ & No follow-up \\
\hline $\begin{array}{l}\text { Urothelial } \\
\text { carcinoma }\end{array}$ & $\begin{array}{l}\text { Urothelial } \\
\text { carcinoma }\end{array}$ & $\begin{array}{l}\text { Urothelial } \\
\text { carcinoma }\end{array}$ & $\begin{array}{l}\text { CT/tumor } \\
\text { infiltration }\end{array}$ & Metastasis & Chemotherapy \\
\hline $\begin{array}{l}\text { Urothelial } \\
\text { carcinoma }\end{array}$ & $\begin{array}{l}\text { Urothelial } \\
\text { carcinoma }\end{array}$ & $\begin{array}{l}\text { Urothelial } \\
\text { carcinoma }\end{array}$ & CT/inflammation & Recurrence & Diverting ostomy, chemotherapy \\
\hline $\begin{array}{l}\text { Urothelial } \\
\text { carcinoma }\end{array}$ & $\begin{array}{l}\text { Urothelial } \\
\text { carcinoma }\end{array}$ & $\begin{array}{l}\text { Urothelial } \\
\text { carcinoma }\end{array}$ & $\begin{array}{l}\text { CT/inflammatory } \\
\text { process }\end{array}$ & Recurrence & Diverting ostomy, chemotherapy \\
\hline $\begin{array}{l}\text { Prostatic } \\
\text { adenocarcinoma }\end{array}$ & $\begin{array}{l}\text { Prostatic } \\
\text { adenocarcinoma }\end{array}$ & $\begin{array}{l}\text { Prostatic } \\
\text { adenocarcinoma }\end{array}$ & $\begin{array}{l}\text { CT/ } \\
\text { lymphadenopathy }\end{array}$ & $\begin{array}{l}\text { N0 staging } \\
\text { to N1 }\end{array}$ & New androgen suppression \\
\hline $\begin{array}{l}\text { Urothelial } \\
\text { carcinoma }\end{array}$ & $\begin{array}{l}\text { Urothelial } \\
\text { carcinoma }\end{array}$ & $\begin{array}{l}\text { Urothelial } \\
\text { carcinoma and prostatic } \\
\text { adenocarcinoma }\end{array}$ & CT/carcinoma & Recurrence & Chemotherapy \\
\hline $\begin{array}{l}\text { Urothelial } \\
\text { carcinoma }\end{array}$ & $\begin{array}{l}\text { Urothelial } \\
\text { carcinoma }\end{array}$ & $\begin{array}{l}\text { Urothelial } \\
\text { carcinoma }\end{array}$ & CT/carcinoma & Recurrence & Chemotherapy \\
\hline $\begin{array}{l}\text { Poorly differentiated } \\
\text { adenocarcinoma }\end{array}$ & None & None & $\begin{array}{l}\text { CT/large lung } \\
\text { mass and metastasis }\end{array}$ & $\begin{array}{l}\text { Metastasis, end } \\
\text { stage cancer }\end{array}$ & Hospice \\
\hline $\begin{array}{l}\text { Squamous cell } \\
\text { carcinoma }\end{array}$ & $\begin{array}{l}\text { Squamous cell } \\
\text { carcinoma }\end{array}$ & $\begin{array}{l}\text { Squamous cell } \\
\text { carcinoma }\end{array}$ & CT/carcinoma & Recurrence & Radiation \\
\hline $\begin{array}{l}\text { Squamous cell } \\
\text { carcinoma }\end{array}$ & $\begin{array}{l}\text { Squamous cell } \\
\text { carcinoma }\end{array}$ & $\begin{array}{l}\text { Squamous cell } \\
\text { carcinoma }\end{array}$ & CT/mass & $\begin{array}{l}\text { Recurrence, T2 } \\
\text { staging to T3 }\end{array}$ & Chemoradiation \\
\hline $\begin{array}{l}\text { Squamous cell } \\
\text { carcinoma }\end{array}$ & $\begin{array}{l}\text { Squamous cell } \\
\text { carcinoma }\end{array}$ & $\begin{array}{l}\text { Squamous cell } \\
\text { carcinoma }\end{array}$ & $\mathrm{CT} / \mathrm{mass}$ & $\begin{array}{l}\text { Recurrence, } \mathrm{T} 2 \\
\text { staging to T3 }\end{array}$ & Chemoradiation \\
\hline $\begin{array}{l}\text { Squamous cell } \\
\text { carcinoma }\end{array}$ & $\begin{array}{l}\text { Consistent } \\
\text { with histology }\end{array}$ & $\begin{array}{l}\text { Squamous cell } \\
\text { carcinoma }\end{array}$ & $\mathrm{CT} / \mathrm{mass}$ & $\begin{array}{l}\text { Recurrence } \\
\text { vulvar carcinoma }\end{array}$ & Radiation \\
\hline $\begin{array}{l}\text { Adenocarcinoma, } \\
\text { gallbladder primary }\end{array}$ & Adenocarcinoma & $\begin{array}{l}\text { Gallbladder } \\
\text { adenocarcinoma }\end{array}$ & CT/drop metastasis & Metastasis & $\begin{array}{l}\text { Add radiation and start chemotherapy } \\
\text { again }\end{array}$ \\
\hline $\begin{array}{l}\text { Carcinoma, breast } \\
\text { primary }\end{array}$ & $\begin{array}{l}\text { Carcinoma, breast } \\
\text { primary }\end{array}$ & $\begin{array}{l}\text { Breast ductal } \\
\text { adenocarcinoma }\end{array}$ & Not available & Metastasis & Enrolled in clinical trial \\
\hline $\begin{array}{l}\text { Adenocarcinoma, } \\
\text { pancreas primary }\end{array}$ & $\begin{array}{l}\text { Adenocarcinoma, } \\
\text { pancreas primary }\end{array}$ & $\begin{array}{l}\text { Pancreatic } \\
\text { adenocarcinoma }\end{array}$ & $\begin{array}{l}\text { CT/colorectal wall } \\
\text { thickening }\end{array}$ & Metastasis & Chemotherapy \\
\hline $\begin{array}{l}\text { Fibrous tissue, } \\
\text { benign epithelium }\end{array}$ & $\begin{array}{l}\text { Benign fibrous } \\
\text { tissue }\end{array}$ & $\begin{array}{l}\text { Colorectal } \\
\text { adenocarcinoma }\end{array}$ & $\begin{array}{l}\mathrm{CT} / \text { colorectal wall } \\
\text { thickening }\end{array}$ & Benign finding & None \\
\hline $\begin{array}{l}\text { Reactive glandular } \\
\text { epithelium }\end{array}$ & Tubular adenoma & $\begin{array}{l}\text { Colorectal } \\
\text { adenocarcinoma }\end{array}$ & $\begin{array}{l}\mathrm{CT} / \text { colorectal wall } \\
\text { thickening }\end{array}$ & T1 tumor & Preservation of anal sphincter \\
\hline $\begin{array}{l}\text { Lymphocytes, } \\
\text { benign epithelium }\end{array}$ & Crohn's disease & $\begin{array}{l}\text { Colorectal } \\
\text { adenocarcinoma }\end{array}$ & Endoscopy/mass & Benign finding & None \\
\hline
\end{tabular}


Table 1 (continued)

\begin{tabular}{|c|c|c|c|c|c|}
\hline $\begin{array}{l}\text { ERUS-FNA } \\
\text { diagnosis }\end{array}$ & $\begin{array}{l}\text { Histologic } \\
\text { diagnosis }\end{array}$ & History of cancer & $\begin{array}{l}\text { Imaging } \\
\text { diagnosis }\end{array}$ & $\begin{array}{l}\text { Clinical } \\
\text { significance }\end{array}$ & Change in treatment \\
\hline $\begin{array}{l}\text { Benign epithelium and } \\
\text { debris }\end{array}$ & $\begin{array}{l}\text { Submucosal } \\
\text { fibrosis }\end{array}$ & $\begin{array}{l}\text { Colorectal } \\
\text { adenocarcinoma }\end{array}$ & Endoscopy/mass & Benign finding & None \\
\hline $\begin{array}{l}\text { Fluid, benign } \\
\text { epithelium }\end{array}$ & Fluid collection & $\begin{array}{l}\text { Colorectal } \\
\text { adenocarcinoma }\end{array}$ & Endoscopy/mass & Benign finding & None \\
\hline Benign epithelium & $\begin{array}{l}\text { Benign colonic } \\
\text { mucosa }\end{array}$ & $\begin{array}{l}\text { Colorectal } \\
\text { adenocarcinoma }\end{array}$ & $\mathrm{CT} /$ recurrence & Benign finding & No follow-up \\
\hline $\begin{array}{l}\text { Fibrous tissue, } \\
\text { benign epithelium, }\end{array}$ & $\begin{array}{l}\text { Inflammatory } \\
\text { polyp }\end{array}$ & $\begin{array}{l}\text { Gastric } \\
\text { adenocarcinoma }\end{array}$ & $\begin{array}{l}\text { CT/wall thickening, } \\
\text { suspicious }\end{array}$ & Benign finding & None \\
\hline Nondiagnostic & $\begin{array}{l}\text { Sarcomatous } \\
\text { carcinoma }\end{array}$ & $\begin{array}{l}\text { Prostatic } \\
\text { adenocarcinoma }\end{array}$ & $\mathrm{CT} / \mathrm{mass}$ & Recurrence & Chemoradiation \\
\hline $\begin{array}{l}\text { Mucin, benign } \\
\text { epithelium }\end{array}$ & $\begin{array}{l}\text { Submucosal } \\
\text { lipoma }\end{array}$ & $\begin{array}{l}\text { Urothelial } \\
\text { carcinoma }\end{array}$ & $\begin{array}{l}\text { Endoscopy/ } \\
\text { submucosal lipoma }\end{array}$ & Nonmalignant & Rectosigmoid resection \\
\hline $\begin{array}{l}\text { Mucinous cyst, } \\
\text { atypical cells }\end{array}$ & None & Ovarian carcinoma & $\begin{array}{l}\text { Endoscopy/cyst in } \\
\text { muscularis propria }\end{array}$ & Recurrence & Chemotherapy \\
\hline $\begin{array}{l}\text { Markedly atypical } \\
\text { epithelial cells }\end{array}$ & $\begin{array}{l}\text { High-grade } \\
\text { squamous } \\
\text { intraepithelial lesion }\end{array}$ & $\begin{array}{l}\text { Anal intraepithelial } \\
\text { high-grade lesion }\end{array}$ & Endoscopy/mass & Recurrence & Follow-up \\
\hline $\begin{array}{l}\text { Markedly atypical } \\
\text { epithelial cells }\end{array}$ & $\begin{array}{l}\text { Radiation } \\
\text { associated atypia }\end{array}$ & $\begin{array}{l}\text { Squamous cell } \\
\text { carcinoma/cervix }\end{array}$ & Endoscopy/mass & Benign finding & None \\
\hline $\begin{array}{l}\text { Markedly atypical } \\
\text { epithelial cells }\end{array}$ & $\begin{array}{l}\text { Colorectal } \\
\text { adenocarcinoma }\end{array}$ & $\begin{array}{l}\text { Colorectal } \\
\text { carcinoma }\end{array}$ & Endoscopy/mass & $\begin{array}{l}\text { Malignant } \\
\text { finding }\end{array}$ & Rectosigmoid resection \\
\hline
\end{tabular}

Among 24 cases with malignant cytology, CT images favored a benign process such as fluid, hematoma, inflammation, and soft tissue mass in 5 cases.

Nineteen aspirated cases were consistent with benign findings (table 2); 18 had concurrent biopsy or subsequent histologic diagnosis. Benign diagnoses were confirmed in 16/18 cases. The histologic diagnoses included endometriosis, submucosal lipoma, submucosal fibrosis, inflammatory polyp, Crohn's disease, perirectal abscess, retrorectal cyst, prolapsed associated changes, cyst fluid, and epithelial cells with focal atypia in a background of mucin. Core biopsies of $2 / 18$ cases were poorly differentiated carcinoma with sarcomatous features in one case and tubular adenoma in the other case. One case missed the follow-up without having a tissue biopsy. Ten of 19 cases with benign cytology had a clinical history of histologically proven carcinoma (6 colorectal adenocarcinomas, 1 gastric adenocarcinoma, 1 prostatic adenocarcinoma, 1 urothelial carcinoma, and 1 ovarian carcino$\mathrm{ma})$.

Among 19 cases with benign cytology, CT images were suspicious for recurrent malignancy in 2 cases, i.e. inflammatory polyp and benign epithelial cells on core biopsy, respectively.
Three cases were diagnosed as markedly atypical epithelial cells suspicious for carcinoma. All three cases had histologic diagnosis. One was adenocarcinoma arising in a tubular adenoma. The second case had a high-grade squamous intraepithelial lesion (AIN III) and inflammatory polyp on core biopsies. The third case had a history of invasive squamous cell carcinoma of the cervix and showed squamous cell atypia due to radiation effects on cytology.

Two cases had a histologically proven history of rectal carcinoid. The FNA of a perirectal mass in one of them was consistent with carcinoid and the second case showed colonic mucosa.

One case was nondiagnostic due to scant cellularity. Tissue biopsy showed tubular adenoma.

We also correlated the size of the lesions and the number of passes.

The number of passes ranged from 1 to 10 (mean = 3.5 passes). The mass size was recorded for 31 cases. The mass lesions measured $0.6-10 \mathrm{~cm}$ in diameter (mean = $3.15 \mathrm{~cm}$ ). The malignant masses ranged from 0.6 to 10 $\mathrm{cm}($ mean $=2.1 \mathrm{~cm})$ and benign masses ranged from 0.9 to $6.8 \mathrm{~cm}($ mean $=3.07 \mathrm{~cm})$. No significant correlation was noted between the number of passes and the size of 
Table 2. Finding in cases with no history of malignancy

\begin{tabular}{|c|c|c|c|c|}
\hline ERUS-FNA diagnosis & Histologic diagnosis & Imaging diagnosis & Clinical significance & Change in treatment \\
\hline $\begin{array}{l}\text { Few spindle cells, benign } \\
\text { colonic mucosa }(n=2)\end{array}$ & $\begin{array}{l}\text { Colitis with prolapsed } \\
\text { associated changes }\end{array}$ & $\begin{array}{l}\text { Endoscopy/submucosal } \\
\text { mass }\end{array}$ & Benign findings & Noninvasive treatment \\
\hline $\begin{array}{l}\text { Mature nucleated and } \\
\text { anucleated squamous cells }\end{array}$ & Abscess & CT/abscess & Benign findings & Abscess drainage \\
\hline $\begin{array}{l}\text { Inflammation and benign } \\
\text { epithelium }\end{array}$ & $\begin{array}{l}\text { Fibrous tissue with } \\
\text { inflammation }\end{array}$ & $\mathrm{CT} / \mathrm{mass}, \mathrm{MRI} / \mathrm{mass}$ & Benign findings & $\begin{array}{l}\text { Conservative surgery of } \\
\text { pelvic mass }\end{array}$ \\
\hline $\begin{array}{l}\text { Prostatic tissue, seminal } \\
\text { vesicles }\end{array}$ & $\begin{array}{l}\text { Prostatic tissue, seminal } \\
\text { vesicles }\end{array}$ & $\begin{array}{l}\text { MRI/rectal wall thickening } \\
\text { favor infection }\end{array}$ & Benign findings & $\begin{array}{l}\text { Mycobacterial and } \\
\text { bacterial culture }\end{array}$ \\
\hline $\begin{array}{l}\text { Few spindle cells mucin, } \\
\text { benign colonic mucosa, }\end{array}$ & Fibroblastic proliferation & $\begin{array}{l}\text { Endoscopy/submucosal } \\
\text { hard mass }\end{array}$ & Benign findings & $\begin{array}{l}\text { Conservative surgery of } \\
\text { pelvic mass }\end{array}$ \\
\hline Benign glandular epithelium & $\begin{array}{l}\text { Endometriosis involving } \\
\text { the bowl }\end{array}$ & Endoscopy/mass & None & Rectosigmoid resection \\
\hline Nondiagnostic & Tubular adenoma & Endoscopy/mass & Staging & Resection \\
\hline Carcinoid & History of carcinoid & $\begin{array}{l}\text { Endoscopy/small } \\
\text { submucosal mass }\end{array}$ & Nonmalignant & No additional work up \\
\hline Benign epithelial cells & History of carcinoid & Endoscopy/lymph node & Benign findings & None \\
\hline
\end{tabular}

the lesions (table 3). Thirty-three aspirations were done by gastroenterologists in an endoscopy unit and 14 aspirations were performed by interventional radiologists in an ultrasound unit. Thirteen patients received prophylactic antibiotic prior to the procedure, i.e. 9 by gastroenterologists and 4 by radiologists. Forty-five specimens were evaluated on site by a pathologist or a cytotechnologist for adequacy. On-site evaluation was not requested by gastroenterologists for 4 procedures including one rectal adenocarcinoma, one fluid collection status post-rectal cancer resection next to the anastomosis site, one tubular adenoma, and one invasive squamous cell carcinoma of the cervix. All 24 malignant cases were adequate.

Imaging studies included CT scans, MRI, and EUS. On imaging studies the cases presented as perirectal masses which were either solid $(n=43)$ or cystic $(n=4)$. Thirty-three cases were designated as a distinct mass and 14 cases as thickening of the intestinal wall. Four cases were cystic on imaging. Cytomorphologic findings included acute and chronic inflammation and debris,
Table 3. Correlation of size of the mass and number of passes

\begin{tabular}{lll}
\hline & $\begin{array}{l}\text { Small masses } \\
(2.5 \mathrm{~cm} \text { or } \\
\text { smaller) }\end{array}$ & $\begin{array}{l}\text { Larger masses } \\
\text { (greater than } 2.5 \mathrm{~cm} \text { ) }\end{array}$ \\
\hline Minimum mass size, cm & 0.6 & 2.9 \\
Maximum mass size, cm & 2.5 & 10 \\
Average mass size, cm & 1.52 & 4.17 \\
Minimum number of passes & 2 & 2 \\
Maximum number of passes & 6 & 10 \\
Average number of passes & 3.1 & 3.0 \\
Total & 12 & 19 \\
\hline
\end{tabular}

anucleated squamous cells consistent with an epidermal inclusion cyst (fig. 3) and, confirmed on histology, benign appearing colonic mucosa, prostatic cells, and seminal vesicles; one was nondiagnostic due to scant cellularity. 


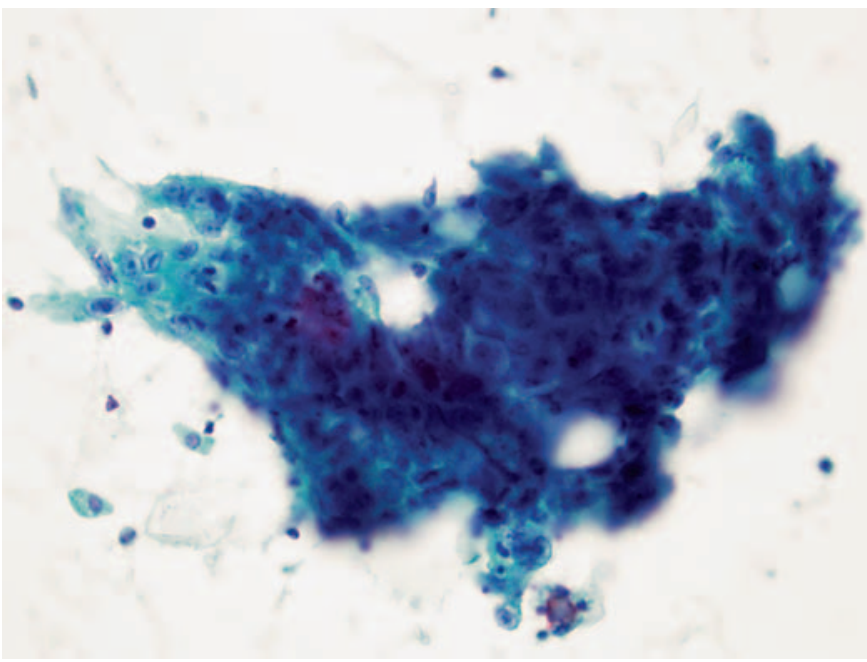

Fig. 1. Rectal adenocarcinoma showing a sheet of malignant cells with hyperchromatic nuclei, irregular nuclear membranes, and prominent nucleoli. Papanicolaou stain. $\times 400$.

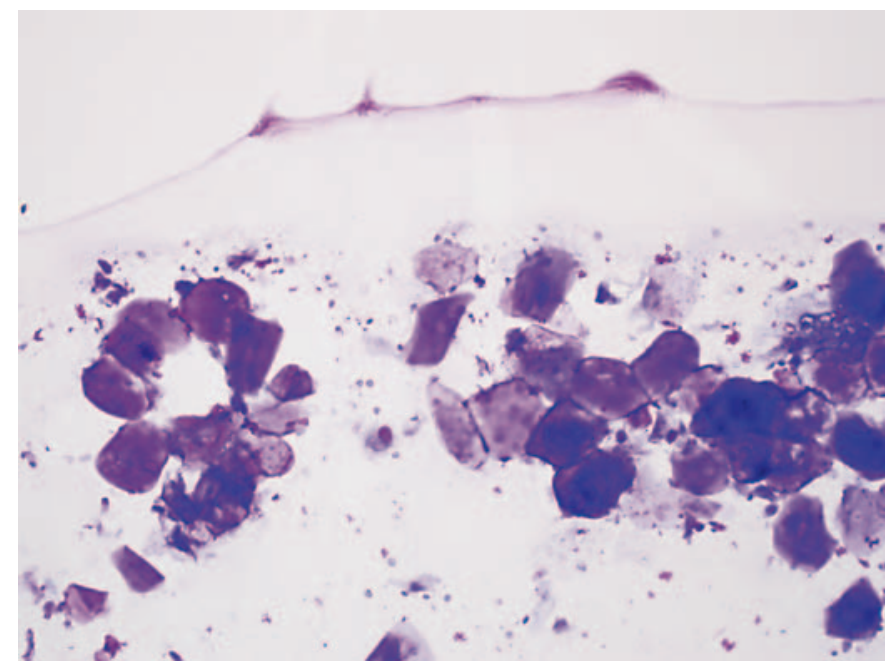

Fig. 3. Epidermal inclusion cyst showing anucleated squamous cells with debris in the background. Diff-Quik stain. $\times 200$.

Three patients had no further follow-up in our institution (one with local recurrence of urothelial carcinoma to perirectal tissue, and two with benign epithelial cells). The third patient had a history of colorectal adenocarcinoma.

All patients tolerated the procedure well and none of them reported any immediate or delayed complications resulting from the procedure.

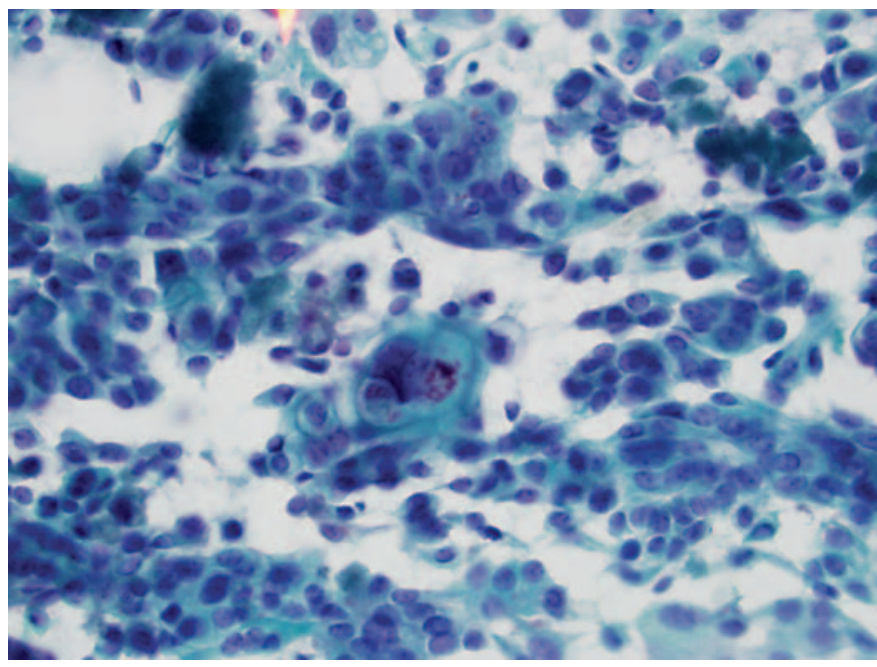

Fig. 2. Urothelial carcinoma with a hypercellular smear consisting of epithelial cells with moderate pleomorphism. Note the extremely large cells in the center. Papanicolaou stain. $\times 400$.

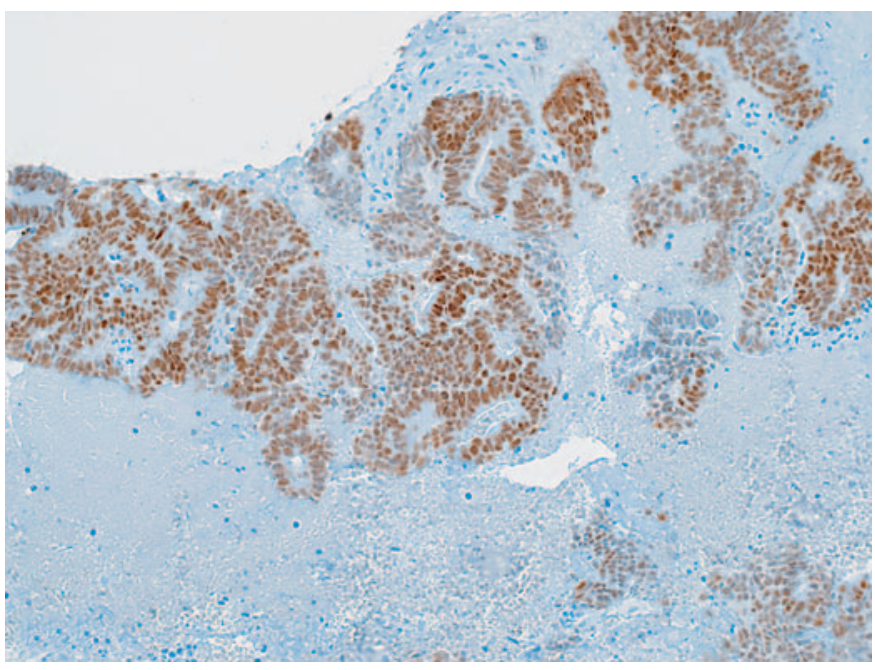

Fig. 4. CDX2 immunostain on a cell block highlights metastatic colorectal carcinoma in a perirectal lymph node. Immunostain. $\times 200$.

\section{Clinical Impact}

ERUS-FNA findings had a significant clinical impact in all cases that were followed up at our institution (table 4). A new diagnosis of metastatic lung adencarcinoma was made in 1 case with no prior history of malignancy. Tumor recurrence was confirmed in 6 cases and tumor metastasis was confirmed in 9 cases. Chemotherapy, ra- 
Table 4. Clinical impact of cytologic diagnosis of perirectal lesions on cancer staging or cytologic confirmation of tumor recurrence in patients diagnosed with cancer

\begin{tabular}{llll}
\hline Primary neoplasms & $\begin{array}{l}\text { Diagnostic } \\
\text { confirmation }\end{array}$ & $\begin{array}{l}\text { Change in } \\
\text { cancer stage }\end{array}$ & Benign diagnosis \\
\hline Colorectal adenocarcinoma $(\mathrm{n}=16)$ & 7 & 3 & 6 \\
Urothelial carcinoma $(\mathrm{n}=7)$ & 5 & 1 & 1 \\
Squamous cell carcinoma $(\mathrm{n}=5)$ & 2 & 2 & 1 \\
Prostatic adenocarcinoma $(\mathrm{n}=1)$ & - & 1 & - \\
Adenocarcinoma of the gallbladder $(\mathrm{n}=1)$ & - & 1 & - \\
Adenocarcinoma of the lung $(\mathrm{n}=1)$ & - & 1 & - \\
Lobular carcinoma of the breast $(\mathrm{n}=1)$ & - & 1 & - \\
Adenocarcinoma of the pancreas $(\mathrm{n}=1)$ & - & - & 1 \\
Gastric adenocarcinoma $(\mathrm{n}=1)$ & - & - & - \\
Ovarian carcinoma $(\mathrm{n}=1)$ & 1 & & \\
\hline
\end{tabular}

diation, or chemoradiation was given to all but 2 cases of carcinoma (one went to hospice and the other had recurrent urothelial carcinoma and had no follow-up in our institution).

Tumor staging for colorectal carcinomas was changed in 8 cases based on cytologic diagnosis of malignancy including T2 to T3 in 4 cases, N0 to N1 in 3 cases, and N0 to $\mathrm{N} 2$ in 1 case. Based on positive findings for malignancy, 4 patients with colorectal adenocarcinoma were treated with chemoradiation followed by surgery. Chemoradiation was started for a patient diagnosed with metastasis from adenocarcinoma of the gallbladder. The amount and field of radiation was changed in one patient due to a change of $\mathrm{T}$ staging from $\mathrm{T} 2$ to $\mathrm{T} 3$. A patient with metastatic breast carcinoma to the perirectal area was started on a clinical trial. The patient with a large lung mass, clinical evidence of widespread metastatic carcinoma, and metastatic lung adenocarcinoma to the perirectal area went to hospice. ERUS-FNA provided adequate diagnostic material on cell block to perform immunostains to confirm recurrence or metastasis (fig. 4).

The rectal sphincter was preserved in a patient with benign diagnosis of perirectal FNA. The patient underwent transanal resection for adenocarcinoma arising in the tubular adenoma. Four cases with no history of malignancy and benign appearing cells on cytology underwent surgery due to presence of mass lesions or severe pain. One had rectosigmoid resection and was diagnosed with a large submucosal lipoma. The second case was a young woman with a prolonged history of abdominal pain, several FNAs and core biopsies with benign findings and imaging highly suspicious for malignancy. She underwent hysterectomy, rectal resection, and numerous pelvic nodule resections. All resected organs were extensively affected by endometriosis. The third case was a 35-year-old woman with a history of severe rectal and abdominal pain, and endometriosis of the umbilicus. She had a rectosigmoid resection and was diagnosed with endometriosis involving the rectal wall with associated serosal adhesions. The fourth case had a large pelvic mass suspicious for a neoplasm on imaging. Fibrous tissue with inflammation was found upon conservative surgery of the pelvic mass. Four of 19 cases with benign cytology had subsequent core biopsies which were negative for tumor in all 4 cases. Finally, of the cases with marked epithelial atypia, one revealed adenocarcinoma arising in tubular adenoma, one showed extensive high grade squamous lesions, and the third case displayed changes consistent with radiation effects.

\section{Statistical Analysis}

Statistical analysis for EUS-FNA showed $87 \%$ sensitivity, $100 \%$ specificity, a diagnostic accuracy of $90 \%$, a positive predictive value of $100 \%$, and a negative predictive value of $77 \%$.

\section{Discussion}

EUS was first introduced to clinical practice in 1980 [4]. ERUS-FNA was first developed in the early 1990s to enhance the diagnostic capabilities of EUS by providing additional pathological findings [5]. Curved linear array echoendoscopes use an electronic transducer and they offer the ability to perform interventional procedures such as FNA under real-time visualization [6]. New tech- 
niques that have contributed significantly to the evolution of ERUS include three-dimensional ERUS [7], highfrequency miniprobes, transrectal ultrasound-guided biopsy techniques, and hydrogen peroxide-enhanced endosonography [8]. ERUS-FNA is usually well tolerated under conscious sedation on an outpatient basis similar to other endoscopic procedures [6]. It can be performed by a gastroenterologist or radiologist either in an endoscopy unit or an ultrasound unit. Transrectal EUS-guided biopsy is a safe and efficient method for tissue sampling of perirectal lesions. The risk of tumor seeding along the biopsy tract is very small, with only rare cases reported in the literature $[9,10]$. Its complication rate is $1-2 \%$, which is similar to that of computed tomography (CT) or percutaneous ultrasound-guided needle aspirations $[9,11]$. The major reported complications are bleeding and infections in cystic lesions. Most reported complications are from EUS-FNA of the upper gastrointestinal tract or pancreas [12].

Bacteremia occurs after ERUS-FNA at a similar rate to that of colonoscopy but it does not warrant the prophylactic administration of antibiotics [13]. In our study, there were some patients who received prophylactic antibiotics. Contraindications are inability to clearly visualize a lesion or a tumor mass or vessel interposed in the path between the needle and the target.

Our study did not find any significant correlation between the number of passes and the size of the mass. However, Jhala et al. [14] reported a mean of 2.5 passes (range 1-9 passes) for small lesions ( $\leq 25 \mathrm{~mm}$ ) and a mean of 4.5 passes (range 1-11 passes) for large lesions (>25 mm) [14]. Specimens obtained could easily be processed for immunohistochemical staining as there were multiple immunostains for 7 of our cases and potentially for molecular analysis.

In Japan, the current indications for EUS-FNA include: differentiating between benign and malignant lesions, staging of cancer, and to establish the endoscopic diagnosis of malignancy before chemotherapy and/or radiation therapy [15]. Perirectal lymph nodes or masses, submucosal masses, and suspected recurrent cancers in and adjacent to surgical anastomoses are potential targets for ERUS-FNA. Sasaki et al. [16] reported an overall success rate of 95.5\% with EUS-FNA to detect benign and malignant lesions. However, the success rate for imaging investigation alone without the histologic findings was $81.8 \%$ [16]. Jhala et al. [14] reported EUS-FNA as a highly effective technique to obtain samples with an overall diagnostic yield of $96 \%$ from both small and large lesions without affecting the sensitivity, specificity, or diagnostic accuracy. Knight et al. [17] reported overall sensitivity, specificity, and positive and negative predictive values of EUS-FNA of $89,79,89$, and $79 \%$. Our study shows a slightly lower sensitivity of $87 \%$ and a higher specificity of $100 \%$, a diagnostic accuracy of $90 \%$, a positive predictive value of $100 \%$, and negative predictive value of $77 \%$.

Accurate staging of rectal and anal carcinomas is crucial for surgical treatment and neoadjuvant chemotherapy planning. Accurate preoperative staging determines both the type of surgery performed and the decision to use preoperative chemoradiation. Inaccurate staging tends to result from overstaging because of associated peritumoral inflammation and difficulty distinguishing malignant from benign reactive lymphadenopathies. In addition, tumor stenosis prevents full staging in approximately $14 \%$ of patients. ERUS-FNA is used in clinical practice to evaluate submucosal lesions, depth of invasion in colorectal cancer, tumor invasion in perirectal fat, and nodal status. The prognosis of rectal cancer correlates with pathological staging at the time of diagnosis. Preoperative chemoradiation in locally advanced rectal cancers improves local control and is associated with an increased rate of sphincter preservation in patients with low-lying cancer [18-20]. In our study, 5 patients had preoperative chemoradiation followed by surgery, and the anal sphincter was preserved in one case with low-lying cancer.

Comparative studies have demonstrated that ERUS is superior to CT in staging accuracy and equivalent to MRI for $\mathrm{T}$ and $\mathrm{N}$ staging. Overall, ERUS accuracy in numerous studies ranges from 80 to $95 \%$ for T staging and 70 to $75 \%$ for $\mathrm{N}$ staging. These levels are slightly higher than the respective $75-85 \%$ for $\mathrm{T}$ staging and $60-70 \%$ for $\mathrm{N}$ staging observed for magnetic resonance imaging (MRI) [21-24] and $65-75 \%$ and $55-65 \%$, respectively, for helical CT [22]. The overall sensitivities and specificities for malignancy reported in the literature range from 82 to $96 \%$ and 94 to $100 \%$, respectively $[5,25,26]$. Preoperative ERUS-FNA may enhance identification of extramesenteric lymph node metastasis outside of the standard radiation field or mesorectal resection margin and may affect the surgical or chemoradiation treatment strategy [27]. ERUS is more accurate to distinguish between benign tumors and invasive carcinomas and between tumors localized to the rectal wall versus tumors with transmural invasion [28]. FNA especially improves the accuracy of endorectal ultrasound in the diagnosis of recurrent rectal cancer [2932]. Restaging after neoadjuvant chemoradiation therapy is compromised by the effects of chemoradiation such as inflammation, edema, necrosis, and fibrosis. Postradiation changes such as fibrosis are difficult to distinguish 
from the residual tumor, and thus poor staging results mainly from overstaging $[1,33]$. ERUS-FNA allows cytologic examination of any suspicious lymph node or intramural mass. ERUS-FNA is also a practical method to approach pelvic masses as discussed in our findings [34, 35].

The limitations of ERUS are that: (1) it is heavily operator dependent, (2) patient acceptability is low particularly in painful conditions [36], (3) it cannot be performed in stenotic tumors, (4) it has limited depth of penetration, and (5) it has a limited field of view [37, 33]. A potential pitfall is overstaging of the tumor. Peritumoral inflammation, hemorrhage, and desmoplastic changes can potentially affect accuracy and result in tumor overstaging [38].

In conclusion, our study illustrates that ERUS-FNA of perirectal lesions is a safe, minimally invasive, and welltolerated procedure that provides valuable information which affects patient management. It is superior to imaging alone in both benign and malignant lesions because it provides cytology material to evaluate the nature of the lesion [30]. It is extremely helpful for accurate staging of colorectal adenocarcinomas and to detect metastatic carcinomas. Immunostains can be performed on cell block material as needed. Benign findings such as inflammation or fibrous tissue can be worked up in a conservative way in cancer-free patients and can prevent aggressive surgery, chemoradiation, or overstaging in cancer patients.

\section{Disclosure Statement}

None.

\section{References}

1 Edelman BR, Weiser MR: Endorectal ultrasound: its role in the diagnosis and treatment of rectal cancer. Clin Colon Rectal Surg 2008;21:167-177.

2 Schaffzin DM, Wong WD: Endorectal ultrasound in the preoperative evaluation of rectal cancer. Clin Colorectal Cancer 2004;4: 124-132.

3 Cartana ET, Parvu D, Saftoiu A: Endoscopic ultrasound: current role and future perspectives in managing rectal cancer patients. J Gastrointestin Liver Dis 2011;20:407-413.

-4 DiMagno EP, Buxton JL, Regan PT, Hattery RR, Wilson DA, Suarez JR, Green PS: Ultrasonic endoscope. Lancet 1980;1:629-631.

5 Williams DB, Sahai AV, Aabakken L, Penman ID, van Velse A, Webb J, Wilson M, Hoffman BJ, Hawes RH: Endoscopic ultrasound guided fine needle aspiration biopsy: a large single centre experience. Gut 1999;44: 720-726.

6 6 Lennon AM, Penman ID: Endoscopic ultrasound in cancer staging. Br Med Bull 2007; 84:81-98.

7 Giovannini M, Bories E, PesentiC, Moutardier V, Lelong B, Delpero JR: Three-dimensional endorectal ultrasound using a new freehand software program: results in $35 \mathrm{pa}$ tients with rectal cancer. Endoscopy 2006; 38:339-343.

$\checkmark 8$ Giovannini M, Ardizzone S: Anorectal ultrasound for neoplastic and inflammatory lesions. Best Pract Res Clin Gastroenterol 2006;20:113-135.
-9 Hirooka Y, Goto H, Itoh A, Hashimoto S, Niwa K, Ishikawa $\mathrm{H}$, Okada N, Itoh T, Kawashima $\mathrm{H}$ : Case of intraductal papillary mucinous tumor in which endosonographyguided fine-needle aspiration biopsy caused dissemination. J Gastroenterol Hepatol 2003; 18:1323-1324.

-10 Shah JN, Fraker D, Guerry D, Feldman M, Kochman ML: Melanoma seeding of an EUS-guided fine needle track. Gastrointest Endosc 2004;59:923-924.

11 Ballo MS, Guy CD: Percutaneous fine-needle aspiration of gastrointestinal wall lesions with image guidance. Diagn Cytopathol 2001;24:16-20.

12 Wiersema MJ, Vilmann P, Giovannini M, Chang KJ, Wiersema LM: Endosonographyguided fine-needle aspiration biopsy: diagnostic accuracy and complication assessment. Gastroenterology 1997; 112: 10871095.

13 Levy MJ, Norton ID, Clain JE, Enders FB, Gleeson F, Limburg PJ, Nelson H, Rajan E, Topazian MD, Wang KK, Wiersema MJ, Wilson WR: Prospective study of bacteremia and complications With EUS FNA of rectal and perirectal lesions. Clin Gastroenterol Hepatol 2007;5:684-689.

14 Jhala NC, Jhala D, Eltoum I, Vickers SM, Wilcox CM, Chhieng DC, Eloubeidi MA: Endoscopic ultrasound-guided fine-needle aspiration biopsy: a powerful tool to obtain samples from small lesions. Cancer 2004; 102:239-246.

- 15 Yamao K, Bhatia V, Mizuno N, Sawaki A, Shimizu Y, Irisawa A: Interventional endoscopic ultrasonography. J Gastroenterol Hepatol 2009;24:509-519.
16 Sasaki Y, Niwa Y, Hirooka Y, Ohmiya N, Itoh A, Ando N, Miyahara R, Furuta S, Goto H: The use of endoscopic ultrasound-guided fine-needle aspiration for investigation of submucosal and extrinsic masses of the colon and rectum. Endoscopy 2005;37:154160.

17 Knight CS, Eloubeidi MA, Crowe R, Jhala NC, Jhala DN, Chhieng DC, Eltoum IA: Utility of endoscopic ultrasound-guided fine-needle aspiration in the diagnosis and staging of colorectal carcinoma. Diagn Cytopathol 2011, E-pub ahead of print.

18 Maor Y, Nadler M, Barshack I, Zmora O, Koller M, Kundel Y, Fidder H, Bar-Meir S, Avidan B: Endoscopic ultrasound staging of rectal cancer: diagnostic value before and following chemoradiation. J Gastroenterol Hepatol 2006;21:454-458.

19 Sauer R, Becker H, Hohenberger W, Rodel C, Wittekind C, Fietkau R, Martus P, Tschmelitsch J, Hager E, Hess CF and others: Preoperative versus postoperative chemoradiotherapy for rectal cancer. N Engl J Med 2004;351:1731-1740.

20 Harewood GC: Assessment of clinical impact of endoscopic ultrasound on rectal cancer. Am J Gastroenterol 2004;99:623-627.

21 Ptok H, Marusch F, Meyer F, Wendling P, Wenisch HJ, Sendt W, Manger T, Lippert H, Gastinger I: Feasibility and accuracy of TRUS in the pre-treatment staging for rectal carcinoma in general practice. Eur J Surg Oncol 2006;32:420-425.

22 Siddiqui AA, Fayiga Y, Huerta S: The role of endoscopic ultrasound in the evaluation of rectal cancer. Int Semin Surg Oncol 2006;3:36. 
23 Meyenberger C, Huch Boni RA, Bertschinger P, Zala GF, Klotz HP, Krestin GP: Endoscopic ultrasound and endorectal magnetic resonance imaging: a prospective, comparative study for preoperative staging and follow-up of rectal cancer. Endoscopy 1995;27:469-479.

24 Friel CM, Cromwell JW, Marra C, Madoff RD, Rothenberger DA, Garcia-Aguilar J: Salvage radical surgery after failed local excision for early rectal cancer. Dis Colon Rectum 2002;45:875-879.

25 Chang KJ, Wiersema MJ: Endoscopic ultrasound-guided fine-needle aspiration biopsy and interventional endoscopic ultrasonography: emerging technologies. Gastrointest Endosc Clin N Am 1997;7:221-235.

-26 Raut CP, Grau AM, Staerkel GA, Kaw M, Tamm EP, Wolff RA, Vauthey JN, Lee JE, Pisters PW, Evans DB: Diagnostic accuracy of endoscopic ultrasound-guided fine-needle aspiration in patients with presumed pancreatic cancer. J Gastrointest Surg 2003;7: 118-126, discussion 127-128.

27 Gleeson FC, Clain JE, Rajan E, Topazian MD, Wang KK, Levy MJ: EUS-FNA assessment of extramesenteric lymph node status in primary rectal cancer. Gastrointest Endosc 2011;74:897-905.
28 Garcia-Aguilar J, Pollack J, Lee SH, Hernandez de Anda E, Mellgren A, Wong WD, Finne CO, Rothenberger DA, Madoff RD: Accuracy of endorectal ultrasonography in preoperative staging of rectal tumors. Dis Colon Rectum 2002;45:10-15.

29 Layfield LJ, Gopez EV: Percutaneous imageguided fine-needle aspiration of peritoneal lesions. Diagn Cytopathol 2003;28:6-12.

30 Hunerbein M, Totkas S, Moesta KT, Ulmer C, Handke T, Schlag PM: The role of transrectal ultrasound-guided biopsy in the postoperative follow-up of patients with rectal cancer. Surgery 2001;129:164-169.

31 Morken JJ, Baxter NN, Madoff RD, Finne $\mathrm{CO}$ 3rd: Endorectal ultrasound-directed biopsy: a useful technique to detect local recurrence of rectal cancer. Int J Colorectal Dis 2006;21:258-264.

32 Woodward T, Menke D: Diagnosis of recurrent rectal carcinoma by EUS-guided fineneedle aspiration. Gastrointest Endosc 2000; 51:223-225.
33 Bhutani MS: Recent developments in the role of endoscopic ultrasonography in diseases of the colon and rectum. Curr Opin Gastroenterol 2007;23:67-73.

- 34 Sailer M, Bussen D, Fein M, Freys S, Debus SE, Thiede A, Fuchs KH: Endoscopic ultrasound-guided transrectal biopsies of pelvic tumors. J Gastrointest Surg 2002;6:342-346.

35 Takahashi Y, Irisawa A, Bhutani MS, Hikichi T, Takagi T, Shibukawa G, Wakatsuki T, Imamura H, Sato A, Sato M, Ikeda T, Suzuki R, Obara K, Hashimoto Y, Watanabe K, Ohira $\mathrm{H}$ : Two cases of retroperitoneal liposarcoma diagnosed using endoscopic ultrasound-guided fine-needle aspiration (EUSFNA). Diagn Ther Endosc 2009;2009: 673194.

36 Saranovic D, Barisic G, Krivokapic Z, Masulovic D, Djuric-Stefanovic A: Endoanal ultrasound evaluation of anorectal diseases and disorders: technique, indications, results and limitations. Eur J Radiol 2007;61: 480-489.

37 Kav T, Bayraktar Y: How useful is rectal endosonography in the staging of rectal cancer? World J Gastroenterol 2010;16:691-697.

- 38 Mackay SG, Pager CK, Joseph D, Stewart PJ Solomon MJ: Assessment of the accuracy of transrectal ultrasonography in anorectal neoplasia. Br J Surg 2003;90:346-350. 